CLINICAL RESEARCH ARTICLE

\title{
Quality assessment and response to less invasive surfactant administration (LISA) without sedation
}

\author{
Ellen de Kort ${ }^{1,2}$, Suzanne Kusters ${ }^{3}$, Hendrik Niemarkt ${ }^{1}$, Carola van Pul ${ }^{4}$, Irwin Reiss ${ }^{2}$, Sinno Simons ${ }^{2}$ and Peter Andriessen ${ }^{1}$
}

BACKGROUND: Although sedative premedication for endotracheal intubation is considered standard of care, less invasive surfactant administration (LISA) is often performed without sedative premedication. The aim of this study was to assess success rates, technical quality and vital parameters in LISA without sedative premedication.

METHODS: Prospective observational study in 86 neonates $<32$ weeks' gestation. LISA was performed according to a standardized protocol without use of sedative premedication. Outcome measures were success rates of LISA attempts, reasons for failure and quality of technical conditions. In 37 neonates, heart rate and oxygen saturation levels from 20 min before until 30 min after start of LISA were collected.

RESULTS: In $48 \%$ of LISAs the first attempt failed and in $34 \%$ quality of technical conditions was inadequate. The success rate was significantly correlated with quality of technical conditions and experience of the performer. Desaturations $<80 \%$ occurred in $54 \%$ of patients while bradycardia $<80 / \mathrm{min}$ did not occur.

CONCLUSION: This study shows a relatively low success rate of the first attempt of LISA, frequent inadequacy of technical quality and frequent oxygen desaturations. These effects may be improved by the use of sedative premedication.

Pediatric Research (2020) 87:125-130; https://doi.org/10.1038/s41390-019-0552-z

\section{INTRODUCTION}

Respiratory distress syndrome (RDS) is one of the major causes of neonatal respiratory morbidity and mortality. ${ }^{1}$ Although the early use of nasal continuous positive airway pressure (CPAP) significantly reduced the need for surfactant replacement therapy in RDS, still over half of all preterm neonates with RDS require surfactant. $^{2}$ Historically, surfactant was administered via an endotracheal tube during a period of mechanical ventilation. As mechanical ventilation may cause ventilator-induced lung injury and increases the risk of bronchopulmonary dysplasia (BPD), ${ }^{3}$ the technique of less invasive surfactant administration (LISA) was developed. In this technique surfactant is administered via a thin catheter in spontaneously breathing infants on nasal CPAP. ${ }^{4}$ A systematic review including six randomized controlled trials with a total of 895 infants showed a significantly reduced incidence of BPD and death and a lesser need of mechanical ventilation in infants treated with LISA compared with infants treated with surfactant via an endotracheal tube during mechanical ventilation. ${ }^{5}$

From endotracheal intubation studies in neonates it is long known that awake laryngoscopy is distressing and painful, and is frequently complicated by a series of serious adverse physiological events. ${ }^{6-16}$ Also, awake intubation increases the time and the number of attempts necessary for successful intubation and increases the risk of traumatic injury to the airway. ${ }^{12,13,17}$ Therefore, in a consensus statement on the prevention and pain in the newborn in 2001 and again by the American Academy of Pediatrics in 2010, it was stated that nonemergency intubation should always be performed with the use of premedication. ${ }^{18,19}$ There is no consensus about the optimal premedication strategy. ${ }^{19}$

Similar to endotracheal intubation, LISA involves the use of a laryngoscope to place the thin catheter between the vocal cords. However, most of the published randomized controlled trials did not use any premedication before LISA. ${ }^{5,20,21}$ Also, in studies investigating LISA practices in different countries, apart from the Nordic countries, LISA is often performed in awake patients. ${ }^{22-24}$ Only in the last 3 years, reports start to appear in the literature using different kinds of premedication prior to LISA. ${ }^{25-28}$ The performance of LISA in awake patients might be considered as a relapse in neonatal medicine. ${ }^{20}$

We performed this observational cohort study to assess the success rate and quality of the technical conditions, and the vital parameter response in preterm newborns undergoing a LISA procedure without sedative premedication.

\section{METHODS}

Study design and patients

We conducted a prospective monocentric observational study on the level III neonatal intensive care unit (NICU) at Máxima Medical Center Veldhoven, the Netherlands, between January 2016 and February 2018. Patients $<32$ weeks' gestation were included in the study if they had RDS and were treated with LISA. We only included every first LISA procedure per patient. LISA for treatment of RDS was implemented into daily practice in our department in 2014 and is since then standard of care. LISA is performed in infants with a gestational age less than 32 weeks with clinical

\footnotetext{
${ }^{1}$ Division of Neonatology, Department of Pediatrics, Máxima Medical Center, Veldhoven, the Netherlands; ${ }^{2}$ Division of Neonatology, Department of Pediatrics, Erasmus UMC -

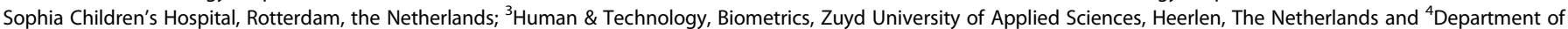
Clinical Physics, Máxima Medical Center, Veldhoven, the Netherlands
}

Correspondence: Ellen de Kort (e.dekort@mmc.nl)

Received: 10 May 2019 Revised: 24 July 2019 Accepted: 16 August 2019

Published online: 27 August 2019 
signs of RDS, respiratory support with nasal CPAP with a positive end-expiratory pressure of at least $6 \mathrm{~cm} \mathrm{H}_{2} \mathrm{O}$, and an oxygen requirement of $30 \%$ or more to maintain $\mathrm{SpO}_{2}$ levels between 88 and $95 \%$.

\section{Description of the LISA procedure}

LISA procedures were performed according to a local standardized protocol. Prior to LISA, patients received a loading dose of caffeine to support the respiratory drive. A dose of atropine $10 \mu \mathrm{g} / \mathrm{kg}$ was administered 5 to $10 \mathrm{~min}$ before start of the procedure to prevent reflex bradycardia during laryngoscopy and catheter placement. About 1 to 2 minutes before start of the procedure, sucrose $20 \%$ $(0.1 \mathrm{ml} / \mathrm{kg})$ was administered in the cheek pouch and facilitated tucking was applied. During the procedure, swaddling was performed to contain the infant and promote comfort. Sedative premedication was not used. A laryngoscope was then used for visualization of the vocal cords and placement of the catheter. At the start of the study we used a 5F umbilical catheter which was placed between the vocal cords with the use of a Magyll forceps. During the study we started to use a shorter and stiffer LISA catheter (Chiesi Pharmaceuticals, Parma, Italy), for which the use of a Magyll forceps was no longer needed. After placement of either catheter, surfactant (Curosurf, 150-200 mg/kg) was instilled over a period of 1-3 min while the patient was spontaneously breathing on nasal CPAP. During surfactant instillation, aspiration was done via the nasogastric tube to check for surfactant spill. After surfactant instillation was complete, the catheter was immediately removed.

\section{Data collection}

We collected the following patient characteristics: gestational age, birth weight, postnatal age at the LISA procedure and gender. Data regarding starting time of the LISA procedure, the quality of technical conditions using a standardized scale, the number of attempts, reasons for failed attempts, and function of the operator of the procedure were collected during and immediately after the procedure on a standardized registration form.

In accordance with the NEAR4KIDS registry definitions of intubation encounters and attempts, ${ }^{29}$ we defined a LISA procedure as one complete procedure of airway management intervention including the administration of surfactant. An attempt was defined as one episode of laryngoscopy, beginning with the insertion of the laryngoscope into the patient's mouth and ending when the laryngoscope was removed. A successful attempt was defined as an episode of laryngoscopy in which the complete amount of surfactant could be administered. An attempt failed if not the complete dose of surfactant could be administered and another laryngoscopy episode was needed to complete surfactant administration. Quality of technical conditions was assessed by the operator of the procedure with the VibyMogensen intubation score. ${ }^{30}$ One missing item was allowed, if more than 1 item of the intubation score was missing, quality of technical conditions could not be judged. Good technical quality was defined as a score on each item $\leq 2$. A score on one or more items $\geq 3$ implied inadequate technical quality.

\section{Vital parameters}

In all infants admitted to the NICU vital parameters are continuously monitored using Intellivue MXI 800 patient monitors (Philips, Hamburg, Germany). All data are saved to a data warehouse system with a sampling rate of $1 \mathrm{~Hz}$. For the purpose of this study, data were extracted from 20 min before until $30 \mathrm{~min}$ after start of the LISA procedure. Data were averaged per minute. Baseline heart rate and oxygen saturation were calculated as median heart rate and oxygen saturation in the period from $20 \mathrm{~min}$ to $10 \mathrm{~min}$ before start of the LISA procedure. The period from 10 min before to start of the LISA procedure was discarded as in this period atropine was administered and nursing handling was needed to install the patient properly for the LISA procedure. Changes in heart rate and oxygen saturation in the $30 \mathrm{~min}$ after the start of LISA in relation to baseline were calculated. Besides this, changes in heart rate and oxygen saturation after the start of LISA compared with baseline were calculated for patients with good versus patients with inadequate technical quality, and for patients with success versus patients with failure of the first attempt.

\section{Statistical analysis}

SPSS (IBM SPSS Statistics for Windows, version $22.0 \mathrm{~m}$ Armonk, NY, USA) was used to analyze the data. Relevant patient data were reported as numbers with percentages for qualitative variables and median and interquartile ranges for quantitative variables. Comparison between groups was performed with the Mann-Whitney $U$ test for continuous variables and the Pearons's Chi square test or Fisher's exact test, as appropriate, for categorical variables. Comparison of vital parameters between baseline and different time points after baseline within the same group of patients was performed with a paired $t$-test.

\section{Ethical approval}

For this observational study we received a waiver for formal ethical approval (Medical Ethical Committee, Máxima Medical Center, Veldhoven, the Netherlands, No. N18.095) according to the Dutch Law of Research with Humans. No additional parental consent was required.

\section{RESULTS}

\section{Study population}

Inclusion of patients is shown in the study flow chart in Fig. 1. During the study period LISA was performed in 111 patients with a GA < 32 weeks. Twenty-five patients were excluded because the standardized registration form was not filled in and therefore data regarding quality of technical conditions and success of LISA attempts were lacking, leaving 86 patients to be included. In 29 patients, data on vital parameters were not available in data warehouse and in 20 patients these data could not be retrieved because the precise starting time of LISA was lacking. Therefore, heart rate and oxygen saturation data from $20 \mathrm{~min}$ before until 30 min after start of LISA were available in 37 patients. Patient characteristics of the study population are shown in Table 1. Patients in whom vital parameter data were lacking, had younger gestational and postnatal ages compared with patients with available data.

Total number of patients with $\mathrm{GA}<32$ weeks treated with LISA $(N=111)$

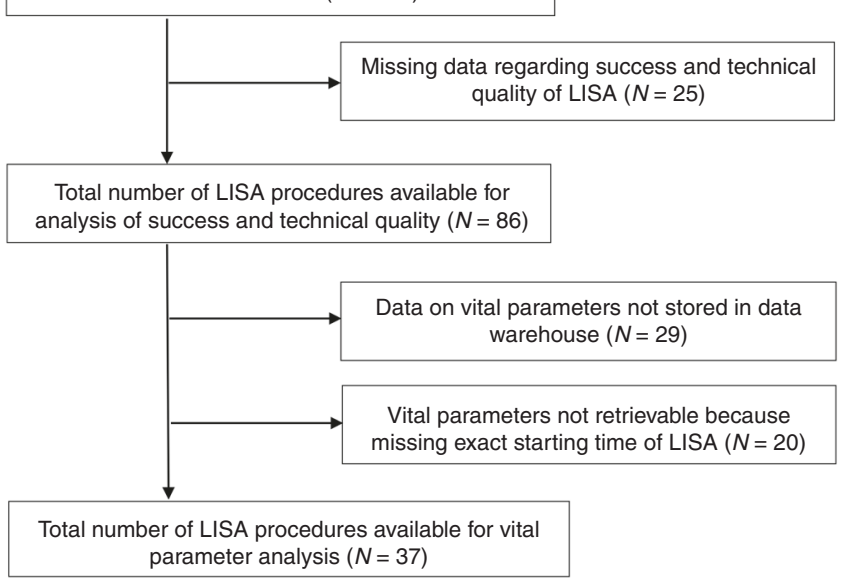

Fig. 1 Study flow chart 
Table 1. Patient characteristics

\begin{tabular}{|c|c|c|c|c|}
\hline & Total study population & Vital parameter analysis & No vital parameter analysis & $P$ value \\
\hline Gestational age (week), median (IQR) & $28.3(26.6-29.7)$ & $29.0(27.1-30.0)$ & $27.9(25.7-29.1)$ & 0.007 \\
\hline Birth weight $<10$ th percentile, $n(\%)$ & $25(29)$ & $13(35)$ & $12(25)$ & 0.34 \\
\hline Postnatal age $(\mathrm{h})$, median (IQR) & $3.3(2.1-8.5)$ & $5.4(2.5-13.2)$ & $2.7(1.9-5.9)^{a}$ & 0.01 \\
\hline
\end{tabular}

Table 2. Patient characteristics and performer experience in relation to the success rate and quality assessment

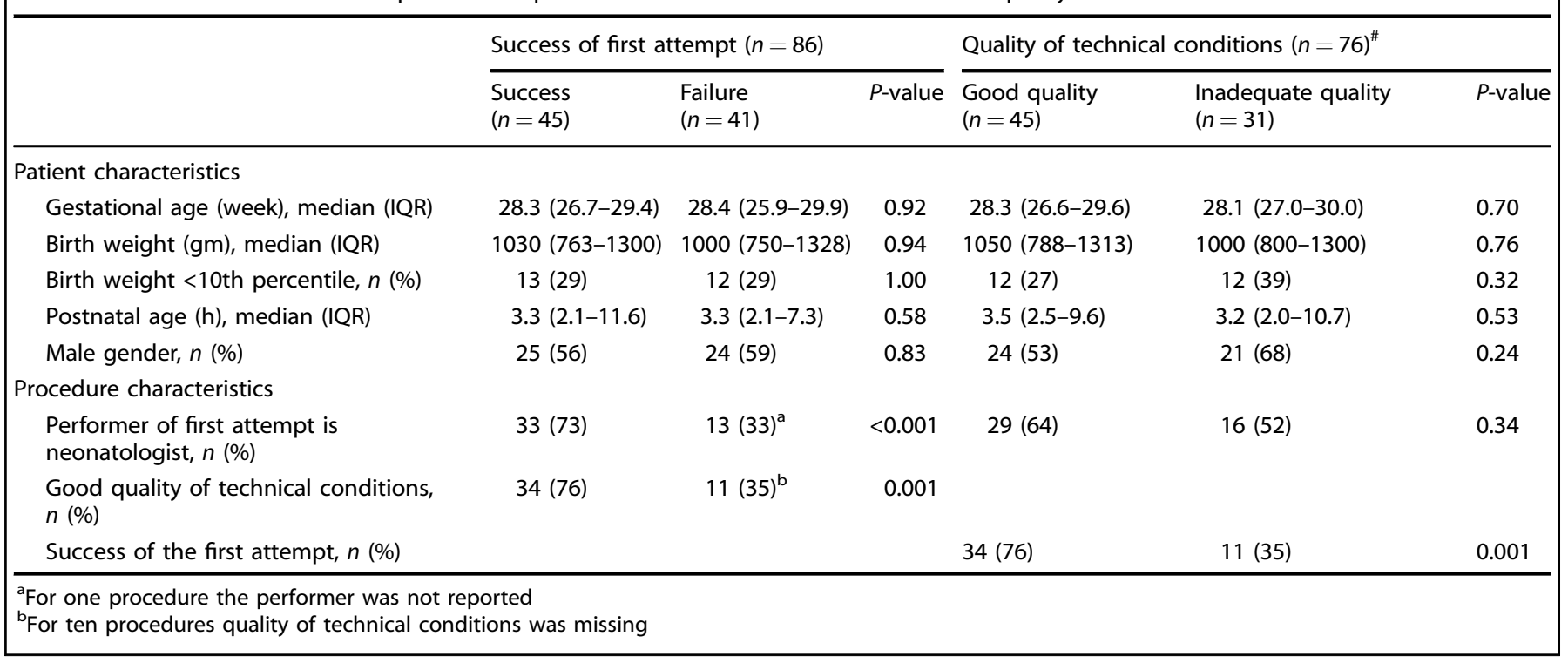

Success of LISA attempts

The LISA procedure was successful at the first attempt in only 45 patients (52\%). In 32 patients (37\%) 2 attempts were required and in 9 patients $(11 \%) 3$ attempts were needed. Reported reasons for failure of first attempts were: inability to visualize the vocal cords in $11(27 \%)$, interruption of the procedure because of significant surfactant spill in $4(10 \%)$, dislocation of the catheter during surfactant administration in $4(10 \%)$, inability to introduce the catheter between the vocal cords in $5(12 \%)$, worsening condition of the patient in $5(12 \%)$, patient resistance in $1(2 \%)$, and other reasons in 4 attempts (10\%). In 7 attempts (17\%) the reason for failure was not reported. The first attempt was performed by a pediatric resident in 7 procedures (8\%), a neonatal nurse specialist in 22 procedures $(26 \%)$, a fellow in neonatology in 10 procedures $(12 \%)$ and a neonatologist in 46 procedures (53\%). Pediatric residents were successful in only 2 attempts (29\%), neonatal nurse specialists were successful in 7 attempts (32\%), fellows in neonatology were successful in 3 attempts (30\%) and neonatologists were successful in 33 attempts $(72 \%)(p=0.003)$.

Table 2 shows patient characteristics and experience of the performer in successful and failed first attempts. These results show there are no statistically significant differences in patient characteristics between LISA procedures in which the first attempt was successful compared with LISA procedures in which the first attempt failed. However, in procedures in which the first attempt was successful, the performer of the procedure was significantly more often a neonatologist than in procedures in which the first attempt failed $(73 \%$ versus $33 \%, p<0.001)$.
Technical quality assessment

Information about the quality of technical conditions was available for 76 LISA procedures (88\%). Quality was good in 45 procedures $(59 \%)$ and inadequate in 31 procedures $(41 \%)$. Table 2 shows the patient characteristics and experience of the performer in procedures with good and with inadequate quality assessment. There were no statistical significant differences in patient characteristics or in the level of experience of the performer between procedures with good and with inadequate technical quality. Quality assessment was, however, related to the success of the first attempt. Of the 45 procedures in which the first attempt was successful, technical quality was good in 34 procedures $(76 \%)$, whereas of the 31 procedures in which the first attempt failed, only 11 procedures (35\%) had good technical quality $(p=0.001)$.

\section{Vital parameters}

Figure 2 shows heart rate and oxygen saturation at baseline and at different time points after start of the LISA procedure. Heart rate significantly increased compared with baseline at all time points with the exception of $t=1 \mathrm{~min}$. Oxygen saturation did not change significantly from baseline at all time points with the exception of $t=1$ and $t=2 \mathrm{~min}$, in which oxygen saturation was significantly lower compared with baseline.

In only two patients (5\%) there was a brief period of bradycardia $<100 /$ min in the first 10 min after start of the LISA procedure. In both patients heart rate restored within $1 \mathrm{~min}$ and never dropped below $80 / \mathrm{min}$. Desaturation were more frequent: in 20 patients 


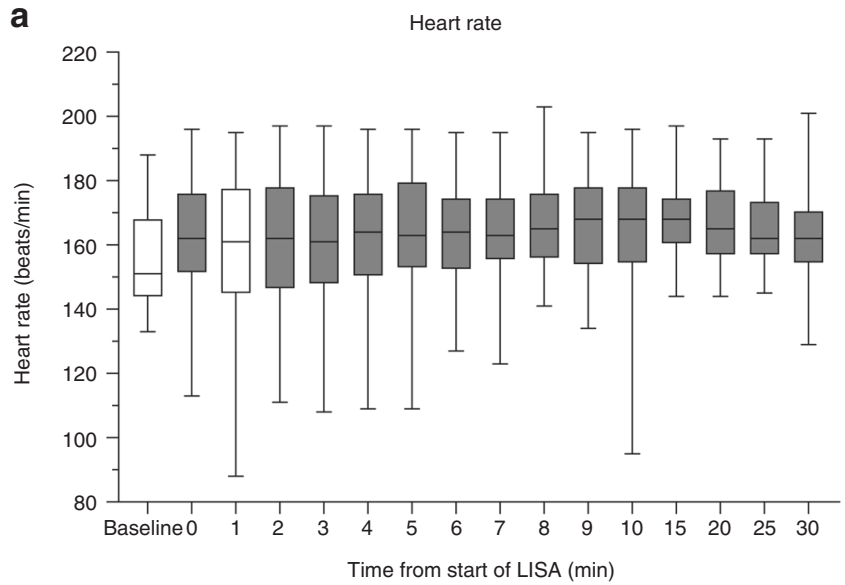

b

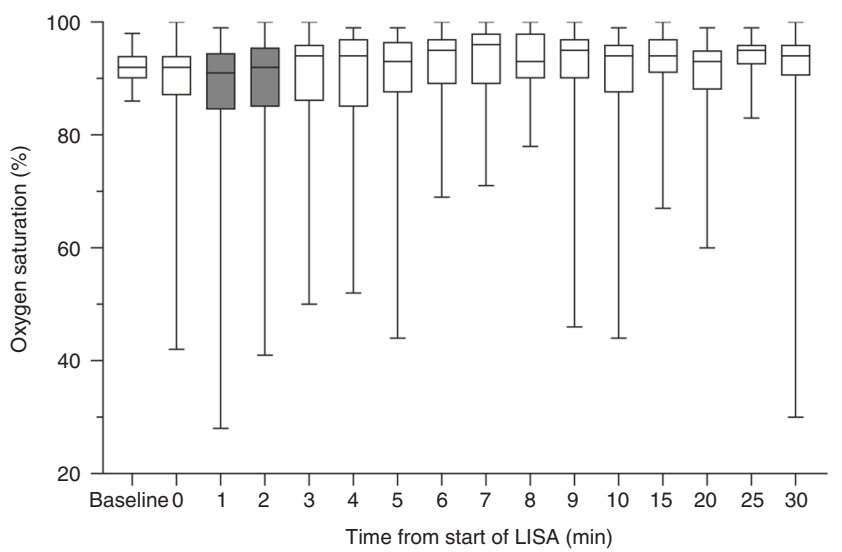

Fig. 2 Heart rate and oxygen saturation at baseline and after start LISA. Dark gray bars indicate significant $(P<0.05)$ differences compared with baseline

(54\%) oxygen saturation dropped below $80 \%$ on 1 or more occasions in the first 10 min after start of LISA.

Figure 3 shows heart rates and oxygen saturation in relation to the success rate of the first attempt and technical quality of each LISA procedure. Heart rate and oxygen saturation did not differ significantly between patients with success versus failure of the first attempt and between good versus inadequate technical quality. There was, however, a significant difference in the time until the deepest drop in oxygen saturation between patients with good versus inadequate technical quality. In patients with good technical quality the lowest oxygen saturation occurred after a median of 2 min after start of LISA versus a median of $5.5 \mathrm{~min}$ for patients in whom the technical quality was inadequate $(p=0.018)$. In patients in whom the first attempts was successful, the deepest drop in oxygen saturation occurred at a median of $4 \mathrm{~min}$, and in patients in whom the first attempt failed the deepest drop in oxygen saturation occurs at median of 2 min. This difference, however, is not statistically significant $(p=0.136)$.

Oxygen desaturations below $80 \%$ occurred in $13 / 19(68 \%)$ of patients with good quality conditions and in $7 / 15(47 \%)$ of patients with inacceptable quality $(p=0.30)$. In procedures with a successful first attempt $(n=19)$, desaturations below $80 \%$ occurred in 13 patients $(68 \%)$, while in procedures in which the first attempt failed $(n=18)$ these desaturations occurred in 7 patients (39\%). This difference was also not statistically significant $(p=0.10)$.

\section{DISCUSSION}

This observational study was performed to assess the quality and effect on vital parameters of LISA procedures performed without the use of sedative premedication. LISA performed in awake patients had a low success rate of the first attempt, and the technical quality frequently was inadequate. Also, there was a significant correlation between failure of the first attempt and the quality of technical conditions, suggesting patient discomfort and intolerance as a cause for first attempt failure. Besides this, there was a high frequency of oxygen desaturations. Combined with the extensive existing evidence on the harmful effects of awake laryngoscopy during endotracheal intubation, our results emphasize the need for better pain reduction and patient comfort during LISA by using sedative premedication.

From 2016 onwards several reports have appeared in the literature using premedication during LISA. Although different strategies of sedative premedication were used, these reports unanimously showed better patient comfort in patients treated with premedication. ${ }^{25-27}$ However, sedative premedication also has adverse effects. In one retrospective study and one randomized controlled trial, propofol as premedication for LISA has shown to increase the need for noninvasive intermittent positive pressure ventilation. ${ }^{25,26}$ Although the need for endotracheal intubation was not increased in patients treated with propofol, the frequency of mechanical ventilation in the first $72 \mathrm{~h}$ of life was higher compared with those that did not receive premedication. ${ }^{20}$ Ketamine as premedication led to a relatively high need for endotracheal intubation. ${ }^{27}$ Since LISA failure causes a higher median number of days on mechanical ventilation, a higher incidence of supplemental oxygen at day 28 and a $20 \%$ lower survival without serious adverse events, ${ }^{31}$ it is important to use sedative premedication that has little to no effect on the respiratory drive in order to prevent LISA failure.

Awake laryngoscopy has considerable effects on vital parameters such as oxygen saturation and heart rate. In our study population, oxygen desaturations $<80 \%$ in the first $10 \mathrm{~min}$ from start of LISA occurred in $54 \%$ of patients. This percentage is lower compared with the studies using premedication prior to LISA. ${ }^{25-28}$ The high incidence of oxygen desaturations in premedicated patients is most probably not due to laryngoscopy, but caused by a pronounced suppression in respiratory drive by the sedative premedication. Besides this, during LISA oxygen desaturations are not only an effect of laryngoscopy but are also caused by the administration of surfactant. The increase in heart rate found in our study is at least partly due to the administration of atropine prior to LISA and is therefore no clear indicator of patient stress and discomfort. The administration of atropine did, however, prevent patients from developing bradycardia compared with the study of Dekker et al. who did not use atropine. ${ }^{26}$

In endotracheal intubation, and presumably also in LISA, the use of premedication can decrease the number of attempts needed for success, ${ }^{12,13,17}$ and improve the quality of technical conditions. ${ }^{32,33}$ In half of all our LISA procedures more than one laryngoscopy episode was needed for completion of the procedure. This success rate was comparable to success rates in studies using premedication prior to LISA, ${ }^{26,27}$ as well as studies using propofol as premedication before endotracheal intubation. ${ }^{34,35}$ Inadequate quality assessment was found in $41 \%$ of procedures. Technical quality seems considerably better when ketamine is used prior to LISA. ${ }^{27}$ To our opinion, the absence of improved success rate after premedication does not mean premedication should not be used. It indicates that we have to do better in premedicated LISA procedures and endotracheal intubations as well.

In summary, comparison of our findings in awake LISA with studies using sedative premedication prior to LISA shows that success rates and effects on vital parameters are comparable. This should not encourage neonatologists to keep on performing LISA 
a

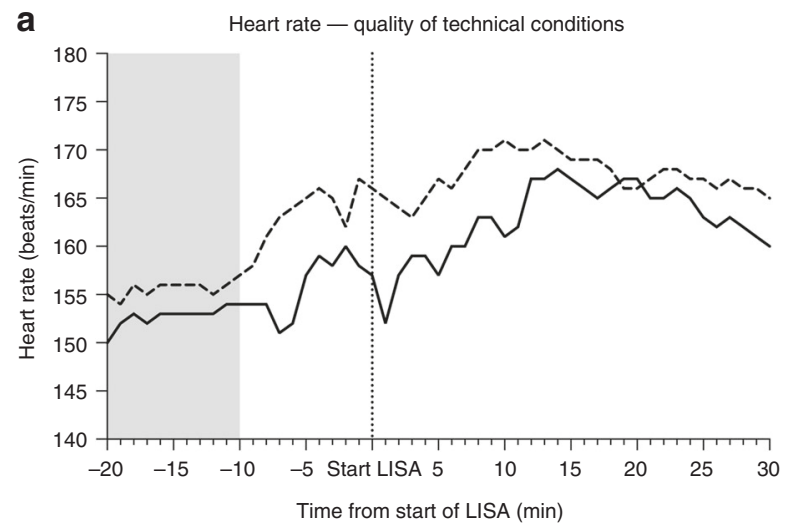

— Good technical conditions ---. Inadequate technical conditions

C

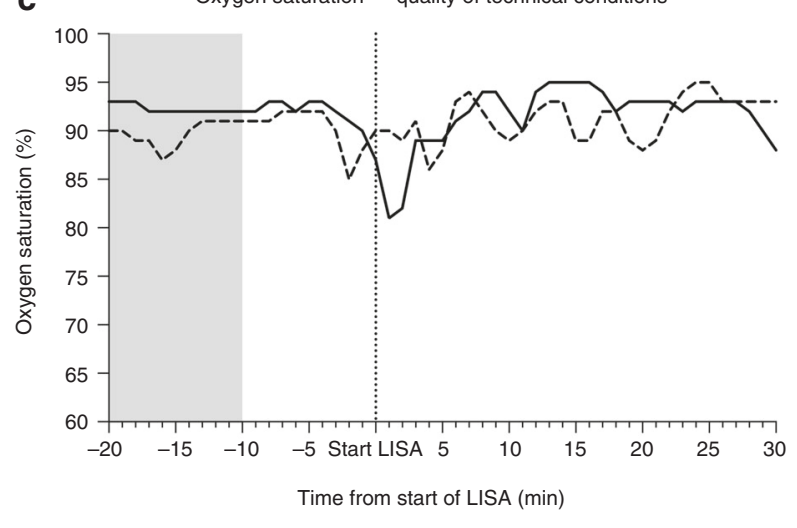

b

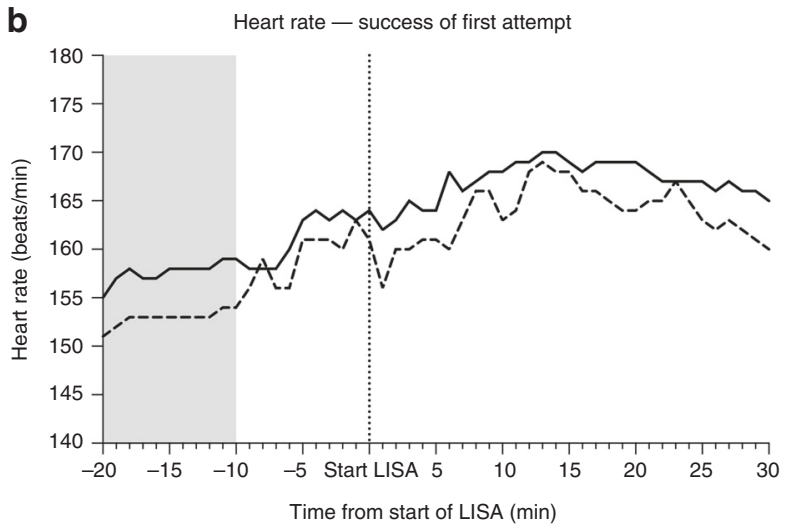

_ Succesful first attempt _--. Failed first attempt

d

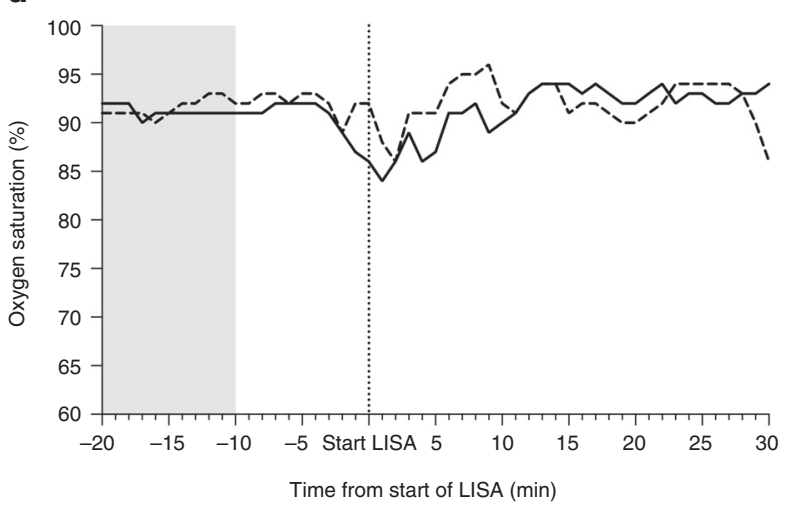

- Succesful first attempt _--. Failed first attempt

Fig. 3 Heart rate and oxygen saturation in relation to the success rate and quality of intubation. Light gray areas indicate the period from which baseline heart rate and oxygen saturation were calculated

in awake patients. There is enough evidence on the harmful effects of awake laryngoscopy during endotracheal intubation and there is no reason to believe these effects would be different in the context of a LISA procedure. LISA, therefore, should always be performed with the use of sedative premedication. This premedication, however, should have the least effect on the respiratory drive and should not hamper LISA success. More research is obviously needed to determine the best premedication strategy.

Success of intubation attempts is not only determined by the use of sedative premedication. Level of experience of the operator is also an important determinant of success. ${ }^{36}$ In our study, we found a significant relation between the success of LISA and the level of experience of the operator. Of all LISA procedures in which the first attempt was successful, it was performed by a neonatologist in $73 \%$, compared with $33 \%$ of the procedures in which the first attempt failed. For endotracheal intubation it is known that the use of premedication improves the success rates of inexperienced operators. ${ }^{37,38}$ It is likely that this is also applicable for LISA. Irrespective of the use of premedication, operator level of experience and number of attempts needed are important factors increasing the odds for endotracheal intubation related adverse events. ${ }^{36,39,40}$ Although there are no studies evaluating the occurrence of LISA related adverse events, it is presumable that the incidence of adverse events during LISA is also influenced by these factors. The operator for LISA should, therefore, be carefully considered.

Lack of data on success and technical quality because of missing registration forms led to the exclusion of almost $25 \%$ of patients that underwent LISA in our study, which could have led to selection bias. The excluded population, however, had comparable baseline characteristics compared with the included patients. The included patients are a good reflection of the total population of preterm infants undergoing LISA and, therefore, our results have good generalizability.

Our study has several limitations. First limitation is the use of atropine prior to LISA. One of the goals of our study was to determine the effects of awake LISA on vital parameters. We found a significant increase in heart rate at all time points after start op LISA, but this is most probably due to the administration of atropine, rather than a reflection of patient stress and discomfort. Nevertheless, we encourage the use of atropine prior to LISA, since it prevents the occurrence of bradycardia and its related risk for hypoxia.

Second limitation is the lack of vital parameter data in $59 \%$ of included patients. Patients with available data had significantly higher gestational and postnatal ages compared with patients in whom vital parameter data were not available. Since we found no influence of gestational and postnatal age on the success rate and technical quality, we would not expect the effects of awake LISA on vital parameters to be different in younger compared with older infants. The availability of vital parameter data in only a small proportion of patients, therefore, will most likely not have affected the validity of our findings. Other limitations are the lack of blood pressure data, which could have helped make a distinction between atropine effect and stress in patients with a significant elevation of heart rate, and the lack of objective measurements of pain and discomfort. 


\section{CONCLUSION}

In our study of LISA procedures performed without sedative premedication, the success rate of the first attempt was only $52 \%$, the technical quality was frequently inadequate, and there was a high incidence of oxygen desaturations. Although providing patient comfort should be a key factor in neonatology, the adverse effects of performing LISA without premedication should be carefully weighed against the negative effects and risks of administering sedative premedication before LISA. Other forms of premedication with lesser effect on the respiratory drive need to be investigated. The use of atropine during LISA resulted in a very low incidence of bradycardia and should therefore be strongly considered, regardless of the use of sedative premedication.

\section{AUTHOR CONTRIBUTIONS}

E.K. conceptualized and designed the study, collected the data, carried out the data analysis and interpretation of the data, drafted the initial paper and reviewed and revised the paper. S.K. was involved in data collection, and reviewed and revised the paper. H.N. participated in the analysis and interpretation of the data, and reviewed and revised the paper. C.P. coordinated and supervised data collection, and reviewed and revised the paper. I.R. reviewed and revised the paper for important intellectual content. S.S. participated in the analysis and interpretation of the data, and reviewed and revised the paper. P.A. was involved in the design of het study, coordinated and supervised data collection, participated in the analysis and interpretation of the data, and reviewed and revised the paper. All authors approved the final paper as submitted and agree to be accountable for all aspects of the work.

\section{ADDITIONAL INFORMATION}

Competing interests: The authors declare no competing interests.

Publisher's note: Springer Nature remains neutral with regard to jurisdictional claims in published maps and institutional affiliations.

\section{REFERENCES}

1. More, K., Sakjuha, P. \& Sha, P. S. Minimally invasive surfactant administration in preterm infants. A meta-narrative review. JAMA Pediatr. 168, 901-908 (2014).

2. Sweet, D. G., Carnielli, G. \& Greisen, G. European consensus guidelines on the management of neonatal respiratory distress syndrome in preterm infants-2013 update. Neonatology 102, 353-368 (2013).

3. Jobe, A. H. The new bronchopulmonary dysplasia. Curr. Opin. Pediatr. 23, 167-172 (2011).

4. Kribs, A. et al. Nonintubated surfactant application versus conventional therapy in extremely preterm infants. A randomized clinical trial. Jama Pediatr. 169, 723-730 (2015).

5. Aldana-Aguirre, J. C., Pinto, M., Featherstone, R. M. \& Kumar, M. Less invasive surfactant administration versus intubation for surfactant delivery in preterm infants with respiratory distress syndrome $>$ a systematic meta-analysis. Arch. Dis. Child Fetal Neonatal Ed. 102, F17-F23 (2017).

6. Marshall, T. A., Deeder, R., Pai, S., Berkowitz, G. P. \& Austin, T. L. Physiologic changes associated with endotracheal intubation in preterm infants. Crit. Care Med. 12, 501-503 (1984).

7. Kelly, M. \& Finer, N. N. Nasotracheal intubation in the neonate. Physiologic responses and effects of atropine and pancuronium. J. Pediatr. 105, 303-309 (1984).

8. Millar, C. \& Bissonnette, B. Awake intubation increases intracranial pressure without affecting cerebral blood flow velocity in infants. Can. J. Anaesth. 41, 281-287 (1994).

9. Stow, P. J., McLeod, M. E., Burrows, F. A. \& Creighton, R. E. Anterior fontanelle pressure responses to tracheal intubation in the awake and anaesthesized infant. Br. J. Anaesth. 60, 167-170 (1988).

10. Friesen, R. H., Honda, A. T. \& Thieme, R. E. Changes in anterior fontanel pressure in preterm neonates during tracheal intubation. Anesth. Analg. 66, 874-878 (1987).

11. Buthada, A., Sahni, R., Rastogi, S. \& Wung, J. T. Randomized controlled trial of thiopental for intubation in neonates. Arch. Dis. Child Fetal Neonatal Ed. 82, F34-F37 (2000).

12. Oei, J., Hari, R., Butha, T. \& Lui, K. Facilitation of neonatal nasotracheal intubation with premedication: A randomized controlled trial. J. Paediatr. Child Health $\mathbf{3 8}$, 146-150 (2002).
13. Barrington, K. J., Finer, N. N. \& Etches, P. C. Succinylcholine and atropine for premedication of the newborn infant before nasotracheal intubation: A randomized, controlled trial. Crit. Care Med. 17, 1293-1296 (1989).

14. Maheshwari, R., Tracy, M., Badawi, N. \& Hinder, M. Neonatal endotracheal intubation: how to make it more baby friendly. J. Paediatr. Child Health 52, 480-486 (2016).

15. Carbajal, R., Eble, B. \& Anand, K. J. S. Premedication for tracheal intubation in neonates: confusion or controversy? Semin. Perinatol. 31, 309-317 (2007).

16. Byrne, E. \& MacKinnon, R. Should premedication be used for semi-urgent or elective intubation in neonates? Arch. Dis. Child 91, 79-83 (2006).

17. Cook-Sather, S. D. et al. A comparison of awake versus paralyzed tracheal intubation for infants with pyloric stenosis. Anesth. Analg. 86, 945-951 (1998).

18. Anand, K. J., International Evidence-Based Group for Neonatal Pain. Consensus statement for the prevention and management of pain in the newborn. Arch. Pediatr. Adolesc. Med. 155, 173-180 (2001).

19. Kumar, P. et al. Premedication for nonemergency endotracheal intubation in the neonate. Pediatrics 125, 608-615 (2010).

20. Tauzin M., Durrmeyer X. Managing neonatal pain in the era of non-invasive respiratory support. Semin. Fetal Neonatal Med. https://doi.org/10.1016/j. siny.2019.04.004 (2019).

21. Kurepa, D., Perveen, S., Lipener, Y. \& Kakkilaya, V. The use of less invasive surfactant administration (LISA) in the united states with review of the literature. $J$. Perinatol. 39, 426-432 (2019).

22. Klotz, D., Porcaro, U., Fleck, T. \& Fuchs, H. European perspective on less invasive surfactant administration-a survey. Eur J. Pediatr. 176, 147-154 (2017).

23. Jeffreys, E., Hunt, K., Dassios, T. \& Greenough, A. UK survey of less invasive surfactant administration. Arch. Dis. Child Fetal Neonatal Ed. 0, F1 (2019).

24. Heiring, C., Jonsson, B., Anderson, S. \& Bjorklund, L. J. Survey shows large differences between the Nordic countries in the use of less invasive surfactant administration. Acta Paediatr. 106, 382-386 (2017).

25. Dekker, J. et al. Sedation during minimally invasive surfactant therapy in preterm infants. Neonatology 109, 308-313 (2016).

26. Dekker, J. et al. Sedation during minimal invasive surfactant therapy: a randomised controlled trial. Arch. Dis. Child Fetal Neonatal Ed. 0, F1-F6 (2018).

27. Bourgoin, L. et al. Administering atropine and ketamine before less invasive surfactant administration resulted in low pain scores in a prospective study of premature neonates. Acta Paediatr. 107, 1184-1190 (2018).

28. Descamps, S. et al. Propofol for sedation during less invasive surfactant administration in preterm infants. Arch. Dis. Child Fetal Neonatal Ed. 0, 1 (2017).

29. Nishisaki, A., Turner, D. A., Brown, C. A.3rd., Walls, R. M. \& Nadkarni, V. M. et al. A national emergency airway registry for children: landscape of tracheal intubation in 15 PICUs. Crit. Care Med. 41, 874-885 (2013).

30. Viby-Mogensen, J. et al. Good clinical research practice (GCRP) in pharmacodynamic studies of neuromuscular blocking agents. Acta Anaesthesiol. Scand. 40, 59-74 (1996).

31. Janssen L., et al. Minimally invasive surfactant therapy (MIST) failure: risk factors and outcome. Arch. Dis. Child. Fetal. Neonatal Ed. https://doi.org/10.1136/ archdischild-2018-316258 (2019).

32. Hassid, S. et al. Randomized controlled trial of sevoflurane for intubation in neonates. Pedia. Anesth. 17, 1053-1058 (2007).

33. Pereira e Silva, Y. et al. Morphine versus remifentanil for intubating preterm neonates. Arch. Dis. Child Fetal Neonatal Ed. 92, F293-F294 (2007).

34. Durrmeyer, X. et al. PRETTINEO research group. Effect of atropine with propofol versus atropine with atracurium and sufentanil on oxygen desaturation in neonates requiring nonemergency intubation. A randomized clinical trial. JAMA 319 (17), 1790-1801 (2018).

35. Smits, A., Thewissen, L., Caicedo, A., Naulaers, G. \& Allegaert, K. Propofol dosefinding to reach optimal effect for (semi-)elective intubation in neonates. J. Pediatr. 179, 54-60 (2016).

36. Foglia E. E., et al. Neonatal intubation practice and outcomes: an international registry study. Pediatrics;143: https://doi.org/10.1542/peds/2018-0902 (2019).

37. Le, C. N. et al. Impact of premedication on neonatal intubations by pediatric and neonatal trainees. J. Perinatol. 34, 458-460 (2014).

38. Dempsey, E. M., Al Hazzani, F., Faucher, D. \& Barrington, K. J. Facilitation of neonatal endotracheal intubation with mivacurium and fentanyl in the neonatal intensive care unit. Arch. Dis. Child Fetal Neonatal Ed. 91, F279-F282 (2006).

39. Foglia, E. E. et al. Factors associated with adverse events during tracheal intubation in the NICU. Neonatology 108, 23-29 (2015).

40. Hatch, L. D. et al. Endotracheal intubation in neonates: a prospective study of adverse safety events in 162 infants. J. Pediatr. 168, 62-66 (2016). 